\title{
Prevention of Contrast Medium Induced Nephropathy by Liposome Encapsulation
}

\author{
Gi-Da Lee, Jyn-Wen Chai, Li-Che Hu, Pei-Hsuan Lu, and Kuo-Chih Liao
}

\begin{abstract}
The study is investigating prospectively the potentials of applying liposome as computed tomography (CT) contrast agent delivery vesicle for prevention of contrast medium induced nephropathy (CIN) incidence both in vitro and in vivo. From dynamic $\mathrm{CT}$ of nude mice, we found that partial encapsulation of $\mathrm{CM}$ in liposome increased the indication of biliary excretion up to 12 folds post $\mathrm{CM}$ administration, and reduced the concentration and duration of $\mathrm{CM}$ accumulation in kidney. From Madin-Darby canine kidney epithelial cell line (MDCK) viability studies, it showed that fully encapsulation of CM in liposome significantly improved the cell viability when exposed to clinical concentration of CM for 24 hours.
\end{abstract}

Index Terms - Liposome, contrast medium, contrast medium induced nephropathy.

\section{INTRODUCTION}

Contrast media (CM) induced nephropathy (CIN) has become the third leading cause of acute renal failure with the increase use for clinical diagnosis and intervention. It is associated with significant risk of morbidity and mortality [1] Although the pathophysiology of CIN has been recognized with renal medulla ischemia and hypoxia resulting renal epithelial/tubular cells damage from toxic effect of CM, the incidence mechanism is still contentious [1]-[3]. The most popular hypothesis of CIN incidence is associated with the impact from hyper-osmolality of CM, however extensive clinical trial studies indicated that the role of osmolality is much lesser and its clinical correlated outcome exhibited in the limited reduction of CIN induced rate when administration with isotonic contrast agents [2]-[4]. Biophysical impacts other than osmolality, such as renal tubular viscosity (increased by $\mathrm{CM}$ ) and renal interstitial pressure (increased by $\mathrm{CM}$ ), or biochemical interaction of $\mathrm{CM}$ metabolites (such as gadolinium ion, releasing from chelating agent of magnetic resonance imaging $\mathrm{CM}$, is a blocker of multiple calcium gated channels) with renal cells might play more important roles in CIN incidence [2]-[7].

Manuscript received April 1, 2013; revised July 11, 2013. This work was supported by grants from the Taichung Veterans General Hospital and National Chung Hsing University (TCVGH-NCHU 1017611) Taichung, Taiwan, Republic of China.

Gi-Da Lee and Jyn-Wen Chai are with the Department of Radiology, Taichung Veterans General Hospital, Taichung City, 40705, Taiwan, Republic of China (e-mail: lee.gida@msa.hinet.net, hubt@mail.vghtc.gov.tw).

Li-Che $\mathrm{Hu}$, Pei-Hsuan Lu, and Kuo-Chih Liao are with the Graduate Institute of Biomedical Engineering, National Chung-Hsing University, 250 Kuo-Kuang Rd., Taichung City, Taiwan, 40227, Republic of China. (e-mail: jackjackjack46@yahoo.com.tw, starwithshadow@hotmail.com, kcliao@dragon.nchu.edu.tw).
Liposome is by far the most successful drug delivery vehicle in commercialization for clinical application [8]. Its long-lasting payload release characteristic has been utilized as anti-cancer, anti-infection and anti-HIV related Kaposi's sarcoma agents, vaccine (hepatitis, influenza), post surgical analgesia, menopause or age-related macular degeneration therapeutics. For chemotherapy, the liposome vesicle can accumulate higher concentration/percentage of drug in tumor (increase therapeutic index), and prevent the drug (toxic agent) exposed to the normal tissue and causing damage during transportation or been sabotaged before arriving target tumor site (reduce side effects). The liposome delivered anticancer agents, such as doxorubicin, have been proved to reduce side effects (cardio-toxicity, hear loss......) while exhibiting superior performance or preserving efficacy in clinical studies with maintaining extended period of above threshold value concentration for treatment [9]-[11]. Considerable efforts have been devoted for the lifetime and integrity of liposomes in the bloodstream for hours to days, increasing the successful rate of transportation to the target location [12], [13]. The enhanced permeability and retention effect (EPR effect) of liposome exhibits passively accumulation of macromolecule on tumor tissue due to the hyper-permeability from tumor neo-vasculature and the lack of lymphatic drainage [14], which contributes in the localization of delivered payload (drug or indicator) in high concentration (up to 177 folds in concentration than administration without liposome [15]).

The application of liposome in delivering $\mathrm{CM}$ is still under laboratory investigations for potentials, and a long road away from commercialization for clinical uses. Those studies focused on utilizing the characteristics of liposome for the following objectives:

1) Permeability of liposome through blood-brain barrier (BBB) can allow early prognosis from brain tumor imaging with $\mathrm{CM}$ before significant compromised BBB in the later stage of cancer development and metastasis [16], [17].

2) Surface modification of liposome with active targeting agents (antibody......etc.) can increase the tumor enhancement of encapsulated CM, such as overcoming the low relaxivity by higher accumulation for MRI [18], or with cell-targeted markers can aid visualization of pathological process [19].

3) High capacity of payload encapsulation and high flexibility of formula or surface modification allow CM included liposome to evolve as multi-model tools for both diagnosis and therapy [19]-[21].

4) The extending circulation lifetime of liposome in vivo allows incorporated $\mathrm{CM}$ for prolonged tumor enhancement or revelation of pathological index [21], [22]. 
Theoretically, delivery of CM with liposome has the potential to prevent the accumulation of $\mathrm{CM}$ in kidney by alternative route of biliary/fecal excretion, undesired exposure of $\mathrm{CM}$ with renal cells, and reduce amount of $\mathrm{CM}$ administrated for maintaining the same contrast effect. The application of liposome in preventing CIN incidence has not been systematically investigated to the authors' knowledge. The study is aiming to investigate prospectively the potentials of applying liposome as computed tomography (CT) contrast agent delivery vesicle for prevention of contrast medium induced nephropathy (CIN) incidence both in vitro and in vivo.

\section{MATERIALS AND METHODS}

\section{A. Dynamic CT of Nude Mice}

NU/NU nude mice were administrated with iobitridol (Xenetix, Guerbert, France), or partially encapsulated iobitridol in liposome (dried lipid film, $10 \mathrm{mg}$ or $20 \mathrm{mg}$, was rehydrated by iobitridol) intravenously as a bolus through the tail vein at the dosage of $1 \mathrm{mg}$ iodine / $\mathrm{g}$. The long circulating liposome was composed of 1,2-dipalmitoyl-sn-glycero-3-phosphatidylcholine (DPPC), L- $\alpha$-phosphatidylcholine (SoyPC), cholesterol (Chol), 1,2-distearoyl-sn-glycero-3-phosphoethanolamine-N-[amino (polyethylene glycol)-2000] in the molar ratio DPPC:SoyPC:Chol:DSPE-PEG2k = 54 :27:16:3 [15].

Dynamic CT imaging was acquired with Philips BR64 (Andover, MA, USA) at following the setting (0.5 sec rotation time, $80 \mathrm{kV}, 80 \mathrm{mAs}, 0.6 \mathrm{~mm}$ beam collimation, $1 \mathrm{~mm}$ slice thickness) at different time points $(0,1,3,8,10,30,60$, 90, 120minutes......etc). Imaging data from the CT scans was transferred to a PACS workstation (Centricity, GE Medical Systems, Milwaukee, Wis, USA) for quantification evaluation. The signal intensity expressed in Hounsfield units (HU) was assessed for the contrasts achieved by CM with or without liposome.

\section{B. Cell Viability of MDCK Cells}

MDCK cells were seeded on 24-well plate to reach $70 \%$ confluence, and then treated with mixture of DMEM medium and iobitrodol or saline (same osmolarity as iobitridol) or column chromatography purified iobitridol encapsulated liposome (distributed in saline with the same osmolarity as iobitridol) in the volume ratio, 1:1, for 24 hours.

Cell viability was quantified by MTT (3-[4, 5-Dimethylthiazol-2-yl]-2,5-diphenyltetrazolium bromide) assay. The yellow solution was reduced by succinate dehydrogenase located in the inner membrane of mitochondria and resulted in the formation of purple crystal, which was dissolved by dimethyl sulfoxide and quantified by absorbance at 570nm with spectrophotometer (Synergy Mx , BioTeK).

\section{RESUlTS AND DISCUSSIONS}

The clinically administrated CMs and their metabolites are primarily excreted through renal excretion due to their hydrophilic characteristics, and with much smaller portion cleared through biliary excretion. Generally, only drugs (and metabolite) with a molecular weight of > $300 \mathrm{~g} / \mathrm{mole}$ and with both polar and lipophilic groups are more likely to be excreted in bile [23]. We found that the presence of liposome had influence on the distribution of CM excretion routes indicated by the signal increase in bladder (waste storage of renal excretion) and intestine (waste storage of biliary excretion) as shown in Fig. 1 and Fig. 2. The partial encapsulation of iobitridol in $10 \mathrm{mg}$ liposome increased the excretion through biliary route up to 12 folds (at 1 hour post administration) and accounted for majority of clearance (>50\%) after 5 hour (Fig. 2).
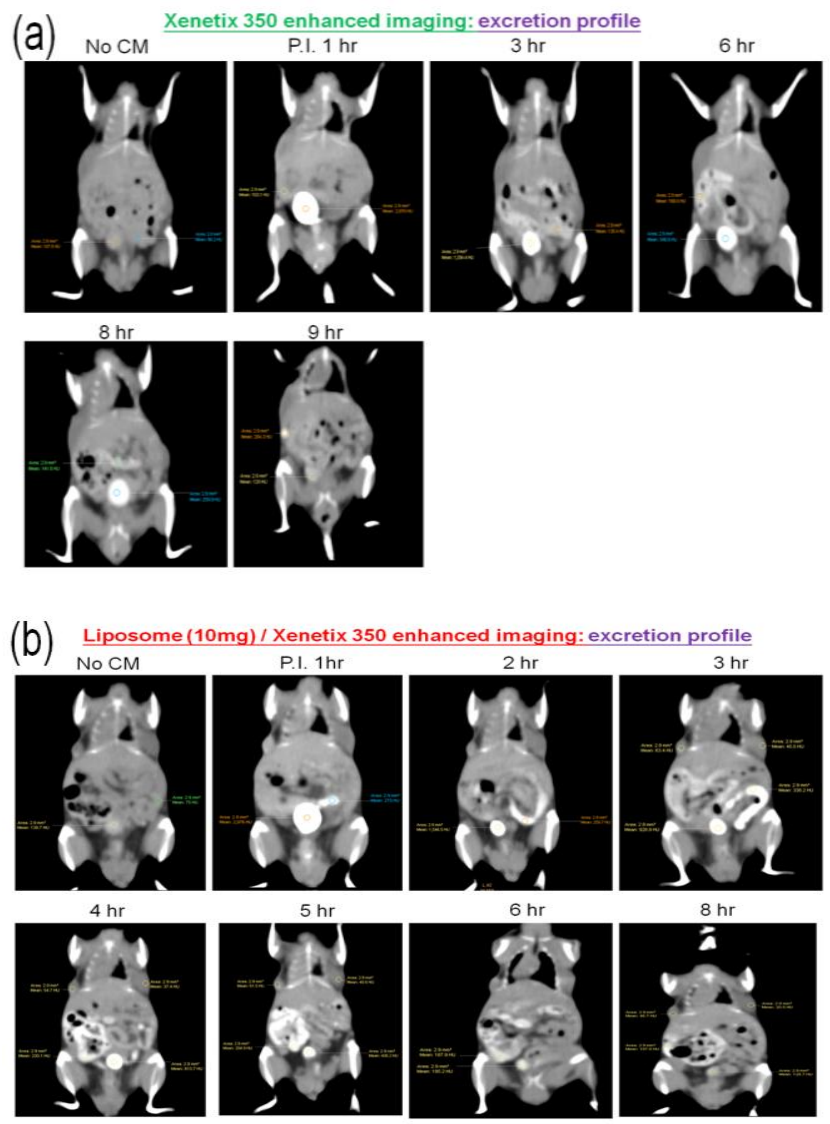

Fig. 1. Contribution of renal and biliary excretion in CM clearance: signal intensity at bladder (renal excretion storage) and intestine (biliary excretion storage) for CM contrasted images (a), and CM/liposome (10mg) contrasted images (b).

In order to investigating the potential dose dependent CIN risk elimination of liposome encapsulation, the accumulation dynamics of $\mathrm{CM}$ in kidney were observed under 3 different contrast enhanced conditions: iobitridol only, iobitridol partially encapsulated with $10 \mathrm{mg}$ liposome and iobitridol partially encapsulated with $20 \mathrm{mg}$ liposome (Fig. 3). We found that the presence of $10 \mathrm{mg} / \mathrm{ml}$ liposome encapsulation reduced the CM signals by $50 \%$ at peak (1 hour post $\mathrm{CM}$ administration), and the higher the dosage of liposome, the quicker the clearance of $\mathrm{CM}$ in kidney. The group with $20 \mathrm{mg} / \mathrm{ml}$ liposome showed no sign of CM presence in kidney after $3^{\text {rd }}$ hour post $\mathrm{CM}$ injection, however the $10 \mathrm{mg} / \mathrm{ml}$ liposome group had CM in kidney up to $5^{\text {th }}$ hour, and pure CM group had CM in kidney lasting more than 7 hours.

In addition to altering the clearance route of $\mathrm{CM}$ and reducing $\mathrm{CM}$ accumulation in kidney, encapsulation by liposome can theoretically prevent the $\mathrm{CM}$ cytotoxicity due 
to the elimination of direct contact / interaction between renal cells and CM, which is shielded by well-proven biocompatible liposome. It is indicated by exposing MDCK cells with medium/iobitridol, or medium/saline (same osmolarity as iobitridol), or medium/column chromatography purified iobitridol encapsulated liposome (distributed in saline with the same osmolarity as iobitridol). The iodine concentration was maintained as $150 \mathrm{mg} / \mathrm{l}$ for both medium/iobitridol and medium/iobitridol/liposome groups to simulating the dilution effect of iobitridol in circulation. The liposome encapsulation had significantly restored the cell viability from $39.8 \%$ to $95.3 \%$ (Fig 4).

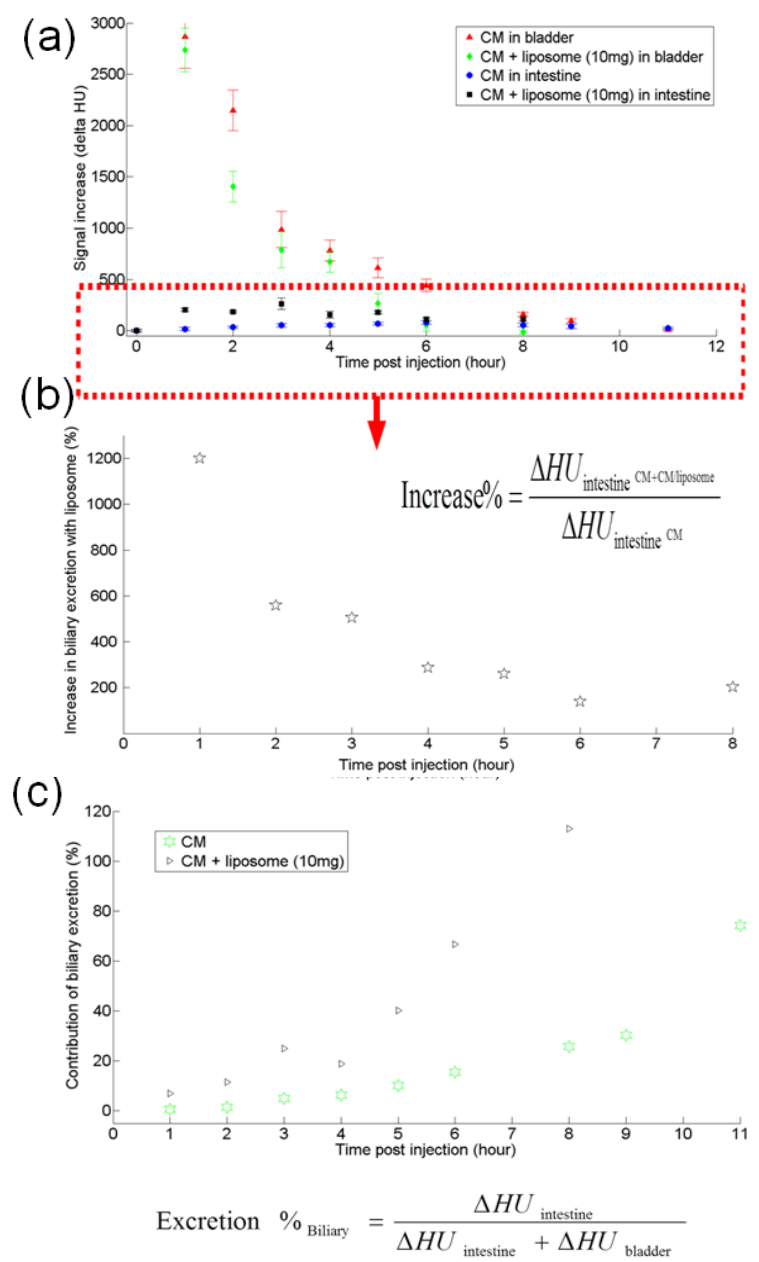

Fig. 2. Contribution of renal and biliary excretion in CM clearance: (a) Excretion dynamics indicated by signal at bladder (renal excretion storage) and intestine (biliary excretion storage). (b) Enhancement of biliary excretion by application of liposome $(10 \mathrm{mg})$. (c) Contribution of biliary excretion in the presence of liposome $(10 \mathrm{mg})$.

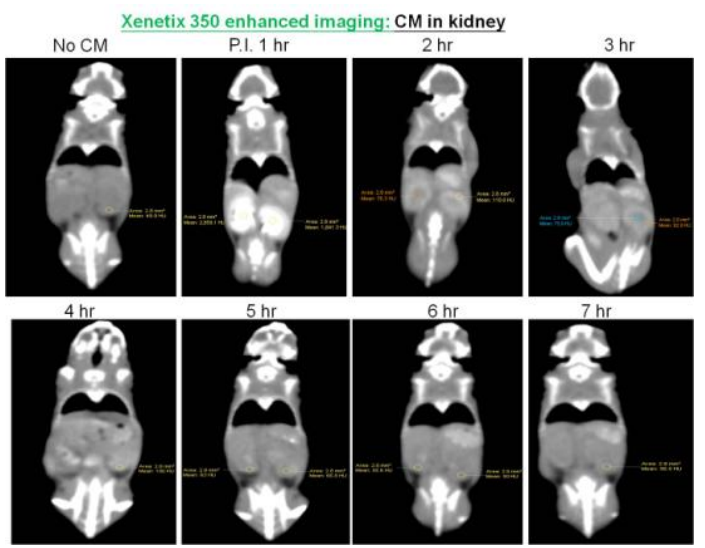

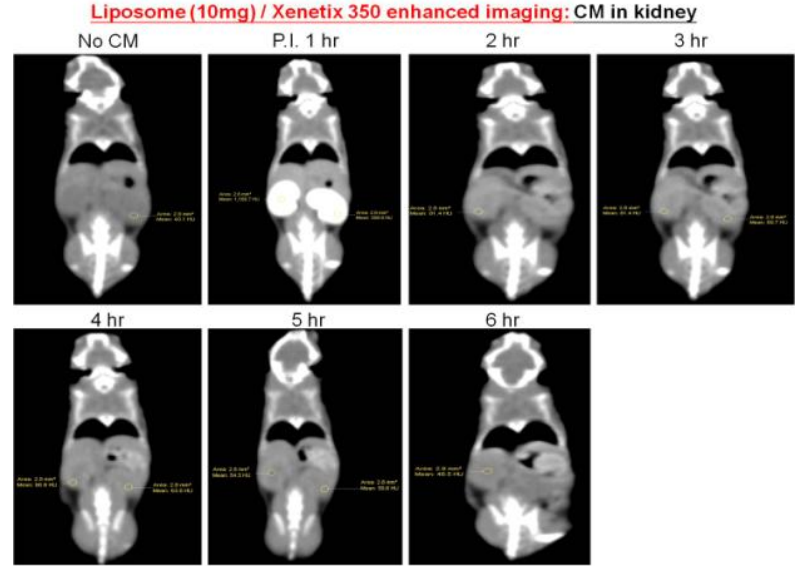

Liposome (20mg) / Xenetix 350 enhanced imaging: CM in kidney

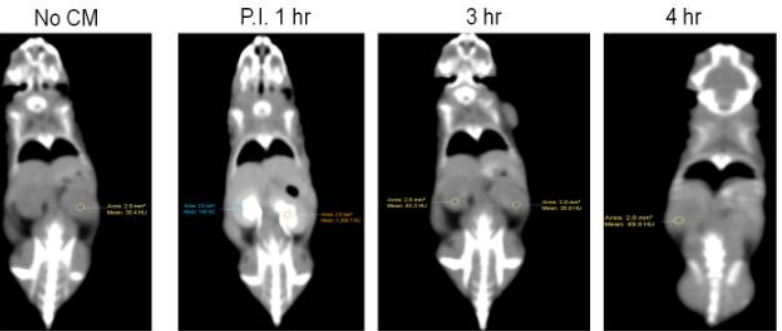

Fig. 3. Accumulation dynamics of CM in kidney under 3 different conditions:iobitridol only, partial encapsulation of iobitridol with $10 \mathrm{mg}$ liposome and partial encapsulation of iobitridol with $20 \mathrm{mg}$ liposome.

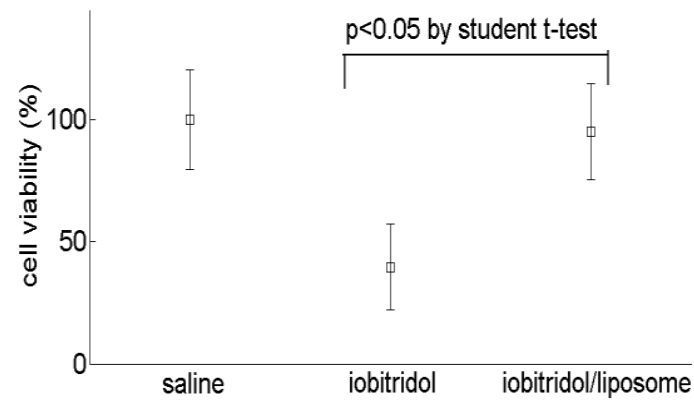

Fig. 4. CM cytotoxicity reduction by liposome encapsulation.

\section{CONCLUSION}

The preliminary results in the study suggested that liposome encapsulation could reduce the risk of CIN by altering the clearance route to avoid accumulation of $\mathrm{CM}$ in kidney (in vivo dynamic CT of mice study) or prevent the direct cytotoxicity of CM on renal cell (MDCK cell viability study).

\section{REFERENCES}

[1] I. Goldenberg and S. Matetzky, "Nephropathy induced by contrast media: pathogenesis, risk factors and preventive strategies," CMAJ, vol. 172, pp. 1461-1471, May 2005.

[2] P. B. Persson, P. Hansell, and P. Liss, "Pathophysiology of contrast medium-induced nephropathy," Kidney Int., vol. 68, pp. 14-22, Jul. 2005.

[3] M. Tepel, P. Aspelin, and N. Lameire, "Contrast-induced nephropathy: a clinical and evidence-based approach," Circulation, vol. 113, pp. 1799-1806, Apr. 2006

[4] R. Solomon, "The role of osmolality in the incidence of contrast-induced nephropathy: a systematic review of angiographic contrast media in high risk patients," Kidney Int., vol. 68, pp. 2256-2263, Nov. 2005

[5] D. R. Martin, R. C. Semelka, A. Chapman, H. Peters, P. J. Finn, B. Kalb, and $\mathrm{H}$. Thomsen, "Nephrogenic systemic fibrosis and contrast agent 
induced nephropathy: risks and benefits of contrast-enhanced MR and CT in renally impaired patients," J. Magn Reson Imaging, vol. 30, pp. 1350-1356, Dec. 2009

[6] J. L. Abraham and C. Thakral, "Tissue distribution and kinetics of gadolinium and nephrogenic systemic fibrosis," Eur J Radiol, vol. 66, pp. 200-207, May. 2008.

[7] M. A. Sieber, H. Pietsch, J. Walter, W. Haider, T. Frenzel, and H.-J Weinmann, "A preclinical study to investigate the development of nephrogenic systemic fibrosis: a possible role for gadolinium-based contrast media," Invest Radiol., vol. 43, pp. 65-75, Jan. 2008.

[8] L. Zhang, F. X. Gu, J. M. Chan, A. Z. Wang, R. S. Langer, and O. C. Farokhzad, "Nanoparticles in medicine: therapeutic applications and developments," Clin Pharmacol Ther., vol. 83, pp. 761-69, May. 2008.

[9] M. E. R. O’Brien, N. Wigler, M. Inbar, R. Rosso, E. Grischke, A. Santoro, R. Catane, D. G. Kieback, P. Tomczak, S. P. Ackland, F. Orlandi, L. Mellars, L. Alland, and C. Tendler, "Reduced cardiotoxicity and comparable efficacy in a phase III trial of pegylated liposomal doxorubicin $\mathrm{HCl}\left(\mathrm{CAELYX}^{\mathrm{TM}} /\right.$ Doxil $\left.^{\circledR}\right)$ versus conventional doxorubicin for first-line treatment of metastatic breast cancer," Ann Oncol., vol. 15, pp. 440-449, 2004.

[10] A. N. Gordon, J. T. Fleagle, D. Guthrie, D. E. Parkin, M. E. Gore, and A J. Lacave, "Recurrent Epithelial Ovarian Carcinoma: A Randomized Phase III Study of Pegylated Liposomal Doxorubicin Versus Topotecan," J Clin Oncol., vol. 19, pp. 3312-3322, Jul. 2001.

[11] O. Lyass, B. Uziely, R. Ben-Yosef, D. Tzemach, N. I. Heshing, M. Lotem, G. Brufman, and A. Gabizon, "Correlation of toxicity with pharmacokinetics of pegylated liposomal doxorubicin (Doxil) in metastatic breast carcinoma," Cancer, vol. 89, pp. 1037-1047, Sep. 2000.

[12] N. D. Santos, C. Allen, A.-M. Doppen, M. Anantha, K. A.K. Cox, R. C. Gallagher, G. Karlsson, K. Edwards, G. Kenner, L. Samuels, M. S Webb, and M. B. Ball, "Influence of poly(ethylene glycol) grafting density and polymer length on liposomes: Relating plasma circulation lifetimes to protein binding," Biochim Biophys Acta, vol. 1768, pp. 1367-1377, Jan. 2007.

[13] A. A. Gabizon, "Liposome circulation time and tumor targeting: implications for cancer chemotherapy," Adv Drug Deliver Rev., vol. 16 , 285-294, Sept. 1995.

[14] R. Duncan, "The dawning era of polymer therapeutics," Nat Rev Drug Discov., vol. 2, pp. 347-360, May 2003.

[15] E. E. Paoli, D. E. Kruse, J. W. Seo, H. Zhang, A. Kheirolomoom, K. D. Watson, P. Chiu, H. Stahlberg, and K. W. Ferrara, "An optical and microPET assessment of thermally-sensitive liposome biodistribution in the Met-1 tumor model: Importance of formulation," $J$ Control Release., vol. 143, pp. 13-22, Dec. 2010.

[16] K. B. Ghaghada, R. R. Colen, C. R. Hawley, N. Pate, and S. Jr. Mukundan, "Liposomal contrast agents in brain tumor imaging," Neuroimaging Clin N Am., vol. 20, pp. 367-378, Aug. 2010.

[17] R. Saito, M. T. Krauze, J. R. Bringas, C. Noble, T. R. McKnight, P. Jackson, M. F. Wendland, C. Mamot, D. C. Drummond, D. B. Kirpotin, K. Hong, M. S. Berger, J. W. Park, and K. S. Bankiewicz, "Gadolinium-loaded liposomes allow for real-time magnetic resonance imaging of convection-enhanced delivery in the primate brain," Exp Neurol., vol. 196, pp. 381-389, 2005.

[18] K. B. Ghaghada, M. Ravoori, D. Sabapathy, J. Bankson, V. Kundra, and A. Annapragada, "New dual mode gadolinium nanoparticle contrast agent for magnetic resonance imaging," PLOS ONE, vol. 4, pp. e7628, Oct. 2009.

[19] W. J. M. Mulder, G. J. Strijkers, J. W. Habets, E. J. W. Bleeker, D. W. J. van der Schaft, G. Storm, G. A. Koning, A. W. Griffioen, and K. Nicolay, "MR molecular imaging and fluorescence microscopy for identification of activated tumor endothelium using a bimodal lipidic nanoparticle," FASEB J., vol. 19, pp. 2008-2010, Oct. 2005.

[20] H. Fattahi, S. Laurent, F. Liu, N. Arsalani, L. Vander Elst, and R. N. Muller, "Magnetoliposomes as multimodal contrast agents for molecular imaging and cancer nanotheragnostics," Nanomedicine (Lond).,vol. 6, pp. 529-544, Apr. 2011.
[21] K. Na, S. A. Lee, S. H. Jung, and B. C. Shin, "Gadolinium-based cancer therapeutic liposomes for chemotherapeutics and diagnostics," Colloids Surf B Biointerfaces., vol. 84, pp. 82-87, May. 2011.

[22] C. T. Badea, K. K. Athreya, G. Espinosa, D. Clark, A. P. Ghafoori, Y. Li, D. G. Kirsch, G. A. Johnson, A. Annapragada, and K. B. Ghaghad, "Computed tomography imaging of primary lung cancer in mice using a liposomal-iodinated contrast agent," PLoS ONE, vol. 7, pp. e34496, Apr. 2012.

[23] X. Yang, Y. A. Gandhi, D. B. Duignan, and M. E. Morris, "Prediction of biliary excretion in rats and humans using molecular weight and quantitative structure-pharmacokinetic relationships," AAPS J., vol. 11, pp. 511-525, Sept. 2009.

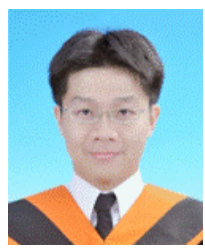

Gi-Da Lee received M. Sc. degree from graduate institute of biomedical engineering, National Chung Hsing University, Taichung, Taiwan in 2011, and B. Sc. degree from medical image and radiological sciences department, Kaohsiung Medical University, Kaohsiung, Taiwan in 2004

$\mathrm{He}$ is currently a radiologic technologist in department of radiology, Taichung Veterans Genera Hospital, Taichung, Taiwan since 2005. He also engaged in clinical research of computed tomography.

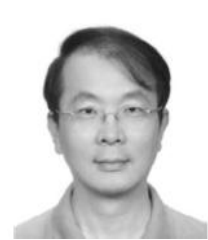

Jyh-Wen Chai received the M.D. and Ph.D. degrees in biomedical engineering from the National Yang- Ming Medical School, Taipei, Taiwan, R.O.C., in 1984 and 2002, respectively.

$\mathrm{He}$ is currently the section chief in the Department of Radiology, Veterans General Hospital, Taichung, Taiwan, and an Associated Professor in the School of Medicine, China Medical University, Taichung, Taiwan. His current research interests include biomedical image processing, computed tomography (CT) and magnetic resonance imaging (MRI).

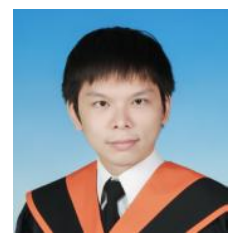

$\mathbf{L i}-\mathbf{C h e} \mathbf{H u}$ is currently working toward M. Sc. degree in graduate institute of biomedical engineering, National Chung Hsing University, Taichung, Taiwan.

$\mathrm{He}$ received B. Sc. degree from biomedical engineering department, YuanPei University, HsinChu, Taiwan in 2011.

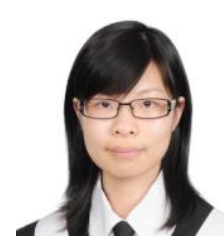

Pei-Hsuan Lu is currently pursuing M. Sc. degree in graduate institute of biomedical engineering, National Chung Hsing University, Taichung, Taiwan.

She received B. Sc. degree from biomedical imaging and radiation science department, China Medical University, Taichung, Taiwan in 2011. Her professional training included internships as Radiologic Technologist in China Medical University Hospital and Taichung Dali Jen-Ai Hospital.

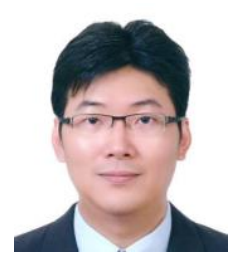

Kuo-Chih Liao received $\mathrm{Ph}$. D. degree from biomedical engineering department, University of Southern California, Los Angeles, California, USA in 2006; M. Sc. (graduate institute of biochemistry, 1997) and B. Sc. (chemical engineering, 1995) degrees from National Taiwan University, Taipei, Taiwan.

$\mathrm{He}$ is an assistant professor at graduate institute of biomedical engineering, National Chung Hsing University, Taichung, Taiwan. His scientific interests focus on application of fiber-optic based platform and nanotechnology for in vivo biomarkers monitoring and therapeutic devices development. 